\title{
Meckel Gruber syndrome: A case report with review of literature
}

\author{
Saime Sundus Uygun ${ }^{1}$, Mesut Sivri ${ }^{2}$, Ahmet Topsakal ${ }^{1}$, Ahmet Hakan Dikener ${ }^{1}$, Hanifi Soylu ${ }^{1}$, Ali Annagur ${ }^{1}$
}

\section{ABST RACT}

Meckel-Gruber syndrome, which is firstly described by Friedrich Meckel and Georg B. Gruber, is an autosomal recessive disorder that is characterized triad of occipital encephalocele, bilateral renal dysplasia and polydactyly. Because of the genetic heterogeneity, multiple organs can be affected. The worldwide incidence varies from 1 in 13,250 to 1 in 140,000 live births. We have reported a male baby born at 37-week gestation, who has occipital encephalocele, polydactyly and cystic dysplasia of both kidneys. As a result of these clinical findings, Meckel-Gruber Syndrome is suspected for his baby. This study is presented to draw attention to the Meckel Gruber Syndrome which has high risk of recurrence and early diagnosis by ultrasonographic screening can be confident.

Keywords: meckel gruber syndrome, encephalocele, renal dysplasia, polydactyly

\section{INTRODUCTION}

Firstly, Friedrich Meckel and Georg B. Gruber have described the Meckel-Gruber syndrome (MGS), which is an autosomal recessive disorder (1). Since the time it was first reported; only 200 cases have been reported until now (2). Meckel-Gruber syndrome is characterized by a combination of renal cysts and variably associated features including developmental anomalies of the central nervous system (typically encephalocele), hepatic ductal dysplasia and cysts, and polydactyly. Rarely this condition can cause renovascular hypertension (3). We report a female baby with a typical triad of Meckel-Gruber syndrome.

\section{CASE REPORT}

A singleton baby was born of a non-consanguineous marriage by $\mathrm{C}$-section at the age of 37 -week gestation. A live male baby weighing $2200 \mathrm{gr}$ was delivered. Antenatal ultrasonography (USG) was suggestive of occipital encephalocele, polydactyly, oligohydramnios and cystic dysplasia of both kidneys. Past and family histories were non-contributory. She did not take any teratogenic drugs. There was no history which was suggestive of an infectious origin for the malformations. In the magnetic resonance examination, there was a mass of size $5 \times 5 \mathrm{~cm}$ with exposed meninges and cerebral tissue around occipital region (Figure 1).

The patient had also agenesis of corpus callosum. All the four limbs showed post-axial polydactyly and syndactyly in both lower limbs. Peripapillary atrophy was found during fundus examination. Abdominal ultrasonography was suggestive of bilateral multicystic dysplastic kidneys (Figure 2).

The baby had also agenesis of corpus callosum. Atrial septal defect was detected at echocardiographic examination. Creatinine and alpha-fetoprotein levels were elevated. He had surgery for repair of an occipital encephalocele at 2 days of age. A genetic analysis could not be done, as the patient was unable to afford this investigation.

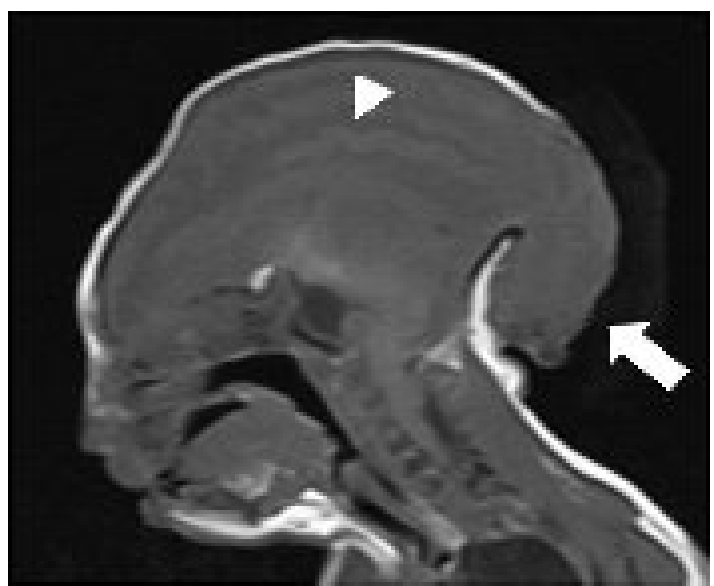

Figure 1: Satittal T1 weighted image shows occipital encephalocele (arrow) and agenesis of corpus callosum (arrowhead)

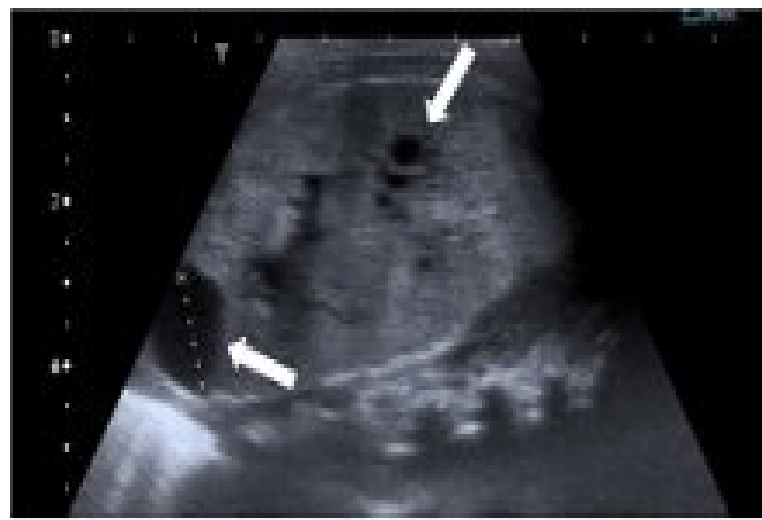

Figure 2: Ultrasonography image shows enlarged echogenic kidney with multiple cysts (arrows). It is compatible with renal cystic dysplasia

\footnotetext{
Department of Neonatology, Selcuk University Faculty of Medicine, Konya, Turkey

Department of Radiology, Selcuk University Faculty of Medicine, Konya, Turkey
}

Received: 15 J une 2015, Accepted: 27 J uly 2015
Correspondence: Ali Annagür, Associate Professor

Department of Neonatology, Selcuk University Faculty of Medicine, Konya, Turkey Phone: +903322244440 


\section{DISCUSSION}

Since the time it was first reported by Meckel in 1822 and subsequently by Gruber in 1934, only 200 cases have been reported (1).

MGS is a multiple congenital malformation syndrome that represents the severe end of the ciliopathy phenotypic spectrum. Ciliopathies are caused by mutations in genes encoding proteins that are components of the primary cilium and basal body. These are multiple organ system disorders caused by dysfunction of the primary cilium, a cytoskeletal appendage which plays essential roles in cellular homeostasis and organ development (4). Besides Meckel-Gruber syndrome, other syndromes with overlapping phenotypes like Bardet-Biedl syndrome or J oubert syndrome also belong to these disorders (5).

MGS is known as the tetralogy of occipital encephalocele, postaxial polydactyly, polycystic kidneys, and hepatic cysts. In Meckel-Gruber syndrome, the incidence of occipital encephalocele and post-axial polydactyly is $60 \%-80 \%$ and $55 \%$ $75 \%$ respectively. Renal disorders are noted in $95-100 \%$ of the cases (6). The other anomalies which form minimal diagnostic criteria include facial abnormalities like cleft lip and palate; urogenital anomalies; ambiguous genitalia; cardiac septal defects; gastro intestinal anomalies like omphalocele and CNS abnormalities like microphthalmia, microcephaly, holoprosencephaly, anencephaly, agenesis of corpus callosum, cerebellar hypoplasia, Dandy-Walker and Arnold-Chiari malformations (7). In most of the cases, hepatic fibrosis, ductal proliferation and cystic dilatation of bile ductile were consistent features. The clinical findings of this syndrome encompass various organ abnormalities as a result of genetic heterogeneity (4). In accordance with the disease, our patient has occipital encephalocele, polydactyly, oligohydramnios and cystic dysplasia of both kidneys. As associated heart anomaly in Meckel-Gruber syndrome is inconstant, our patient has atrial septal defect.

MGS is usually diagnosed prenatally during the second trimester by ultrasound. The classic triad was solely seen in cases diagnosed before the 14th week of gestation. Later in the pregnancy, severe oligohydramnios makes it more difficult to establish the diagnosis by ultrasound alone (8). Alpha fetoprotein in the maternal serum usually elevates. If diagnosis is done by prenatal ultrasound examination termination of pregnancy can be done. When available, autopsy and genetic analysis are gold standard for diagnosis $(2,7)$. If the baby was born alive, brain MRI can detect central pathology, such as

\section{REFERENCES}

1. J ha T, Bardhan J, Das B, Patra KK, Dhali B, Seth S. Meckel-Gruber syndrome: a rare clinical entity. J Indian Med Assoc. 2010;108:611-2.

2. Myageri A, Grampurohit V, Rao R. Meckel Gruber Syndrome: Report of two cases review of literature. J Family Med Prim Care2013; 2:106-18.

3. Moses $M$, Ranger $A$, Yazdani A. Acute Cranial Decompression in Meckel-Gruber Syndrome and SlitVentricle Syndrome with Craniocephalic Disproportion. The J ournal of Craniofacial Surgery. 2011;22:2288-91.

4. Shaheen R, Ansari S, Mardawi EA, Alshammari MJ, Alkuraya FS. Mutations in TMEM231 cause Meckel-Gruber syndrome. J Med Genet. 2013;50:160-2.

5. Eckmann-Scholz C., J onat W., Zerres K., Ortiz-Brüchle $N$. Earliest ultrasound findings and description of splicing mutations in Meckel-Gruber syndrome. Arch Gynecol Obstet. 2012;286:917-21. encephalocele, more detailed. And also abdominal ultrasonography can be used for examining renal pathologies. Our patient was detected by prenatal ultrasound examination at second trimester, family did not accept termination. After birth brain MRI, abdominal ultrasonography was made for further evaluation.

MGS is inherited in an autosomal recessive pattern with six genes described to date. These are 17q21-24 (MGS1), 11q13 (MGS2), 8q21.3-q22.1 (MGS3), 12q21.31-q21.33 (MGS4), $16 q 12.2$ (MGS5), and 4p15.3 (MGS6). This mapping suggests genetic heterogeneity in MGS. Although MGS has genetic heterogeneity and phenotypic variability, the genotypephenotype correlation is not known exactly (9). The worldwide incidence varies from 1 in 13250 to 1 in 140000 live births. As MGS has a high risk (25\%) of recurrence; parents should be informed about the future pregnancies (2).

MGS has to be differentiated from other disorders like trisomy 13 (holoprosencephaly, cleft lip/palate, congenital heart diseases and polydactyly), trisomy 18 (choroid plexus cyst, congenital heart/ kidney disease, rocker bottom feet and polydactyly), Joubert Syndrome (hypoplasia/dysplasia of vermis, facial abnormalities, cystic renal diseases, polydactyly and cleft palate), Bardet-Biedl syndrome (vision loss, mental retardation, renal diseases, polydactyly and cleft palate), and Smith-Lemli-Opitz syndrome (microcephaly, mental retardation and polydactyl). The complexity of the differential diagnosis underlines the need for molecular testing for this syndrome (8, 11).

The prognosis is poor. Unfortunately, there is no effective treatment or cure for MGS (10). The mortality ratio is $100 \%$ Most infants are stillborn or die after postnatal hours or days (8). Gazioglu et al. reported an unusual case of Meckel-Gruber syndrome with a good outcome initially with appropriate treatment and the infant dying at 7 months of age. Death is mainly due to pulmonary hypoplasia (12).

\section{CONCLUSION}

MGS is a lethal and rare autosomal recessive condition characterized by occipital encephalocele, polycystic kidneys, and post axial polydactyly. USG and maternal serum alpha fetoprotein level estimation can be used to learn the recurrence in subsequent gestation periods. Our aim in this review is to enhance the knowledge and spread awareness about this rare and lethal anomaly.
6. Roy J, Pal M. Meckel Gruber Syndrome. J ournal of Clinical and Diagnostic Research. 2013; 7:2102-3.

7. Devecioğlu C, Özdoğan H, Yokuş B. Meckel - Gruber Sendromu: Olgu Sunumu. Dicle Tip Dergisi. 2004;31:658.

8. Alexiev BA, Lin X, Sun CC, Brenner DS. Meckel-Gruber syndrome: pathologic manifestations, minimal diagnostic criteria, and differential diagnosis. Arch Pathol Lab Med. 2006; 130: 1236-8.

9. Szymanska K, Berry I, Logan CV, et al. Founder mutations and genotype phenotype correlations in MeckelGruber syndrome and associatedciliopathies. Cilia. 2012; 1: 18-26.

10. Bolineni C, Nagamuthu EA, Neelala N. Fetal and Pediatric Pathology. 2012; 32:387-93.

11. Panduranga C, Kangle R, Badami R, Patil PV. MeckelGruber syndrome: Report of two cases. J Neurosci Rural Pract. 2012;3:56-9. 
12. Gazioğlu N, Vural M, Seçkin MS, et al. Meckel-Gruber syndrome. Childs Nerv Syst. 1998; 14:142-5.

$\diamond \diamond \diamond \diamond \diamond \diamond \diamond$

http:// www.ejgm. org 